\title{
MEDIDAS OROFACIAIS EM ADOLESCENTES DO ESTADO DO RIO DE JANEIRO SEGUNDO A TIPOLOGIA FACIAL
}

\author{
Orofacial measurements in teenagers \\ from Rio de Janeiro State according to facial typology
}

Sheila Pereira de Castro Guedes ${ }^{(1)}$, Bárbara Veiga Teixeira ${ }^{(2)}$, Débora Martins Cattoni ${ }^{(3)}$

\begin{abstract}
RESUMO
Objetivo: verificar se há diferença entre as medidas orofaciais segundo o tipo facial. Métodos: 39 adolescentes leucodermas entre 15 e 17 anos, de ambos os sexos, com dentição permanente e sem queixa ou histórico de tratamento fonoaudiológico. Utilizando um paquímetro digital CE ELECTRONIC mensurou-se a altura do lábio superior, inferior e filtro, terços superior, médio e inferior da face, assim como distância entre canto externo do olho e comissura labial em ambos os lados. Resultados: considerando a variável tipologia facial houve diferença para a altura do lábio superior, filtro e terços superior e inferior da face. Não se observou diferença entre os tipos faciais para as medidas de altura do lábio inferior, terço médio da face e distância do canto externo do olho à comissura labial, em ambos os lados. Conclusão: na amostra estudada, a altura de lábio superior foi maior no dolicofacial que no mesofacial, a altura do filtro foi maior no dolicofacial que no braquifacial, o terço superior da face foi maior no mesofacial que nos demais tipos e o terço inferior foi maior no dolicofacial que no braquifacial.
\end{abstract}

DESCRITORES: Face; Antropometria; Medidas; Adolescente; Circunferência Craniana; Morfologia

\section{INTRODUÇÃO}

Os critérios para avaliação da tipologia facial dentro da normalidade advêm do processo de crescimento do esqueleto craniofacial, das transformações físicas consequentes e de um processo morfogênico. $O$ crescimento craniofacial acontece por meio de características genéticas de cada indivíduo

(1) Fonoaudióloga; Clínica Odontológica Integrada Ltda, Cabo Frio, RJ; Clínica Médica Santa Maria, São Pedro da Aldeia, RJ; Especializanda em Motricidade Orofacial no CEFAC Pós-Graduação em Saúde e Educação.

(2) Fonoaudióloga; Prefeitura Municipal de Armação dos Búzios, RJ; Clínica Odonto, Búzios, RJ; Clínica OrthoFace, Cabo Frio, RJ; Especializanda em Motricidade Orofacial no CEFAC - Pós-Graduação em Saúde e Educação.

(3) Fonoaudióloga; Professora do CEFAC - Pós-Graduação em Saúde e Educação, São Paulo, SP; Especialista em Motricidade Orofacial pelo CEFAC - Pós-Graduação em Saúde e Educação; Especialista em Saúde Coletiva pela Faculdade de Medicina da Universidade de São Paulo; Mestre em Ciências pela Faculdade de Medicina da Universidade de São Paulo; Doutora em Ciências da Reabilitação pela Faculdade de Medicina da Universidade de São Paulo.

Conflito de interesses: inexistente e os traços faciais são obtidos por hereditariedade, mas podem ser adquiridos ou atenuados por uso ou desuso ${ }^{1-3}$.

Assim, a tipologia facial é a variação da forma do esqueleto craniofacial, resultando nos diversos tipos faciais - longo, médio, curto - cujas características musculares e funcionais são inerentes ao domínio da direção de crescimento ${ }^{2}$. As forças e os fatores do crescimento podem agregar-se a estes padrões verticais da face com as variações do perfil. Os tipos de face reservam padrões diferenciados da musculatura e do desempenho das funções orais distintas dadas suas características esqueléticas ${ }^{2,3}$.

A face é descrita em termos verticais como face longa ou dolicofacial, quando a cabeça é ovalada, comprida e estreita no sentido horizontal com certa tendência à retrusão mandibular. Os sujeitos que apresentam esse tipo facial possuem musculatura elevadora da mandíbula mais delgada e o tipo mais comum de má oclusão é a mordida aberta esquelética. Em contraste, tem-se a face curta ou braquifacial, na qual a cabeça é mais arredondada, mais curta e ampla no sentido horizontal e o complexo nasomaxilar posiciona-se mais posteriormente. 
Os indivíduos com esse tipo facial possuem musculatura elevadora da mandíbula espessa e sua inserção no corpo da mandíbula é ampla e tem tendência a presença de sobremordida. Enquanto a face média ou mesofacial é caracterizada como tendo o padrão de crescimento equilibrado e com boa relação entre os maxilares, normalmente apresentando arco dentário oval ou médio ${ }^{3,4-6}$

Como a avaliação da morfologia facial pode ser realizada de diversas formas, alguns autores sugerem a antropometria enquanto ciência que estuda as mensurações das diferentes partes do corpo, como tamanho, peso e proporções, por meio de uma série de medidas da cabeça e da face ${ }^{7-11}$

A antropometria por sua vez, apresenta vantagens para a avaliação da morfologia do complexo craniofacial. É um método confiável para obter a representação objetiva da cabeça e da face, o que aumenta a confiabilidade da avaliação da morfologia craniofacial. Sugere-se que seja aplicado sempre pelo mesmo examinador. Utiliza-se de técnica simples, objetiva, sem risco e de baixo custo. $\mathrm{Na}$ antropometria direta, a avaliação morfológica é conseguida a partir da utilização do paquímetro ou fita métrica, obtendo-se as medidas diretamente na face do sujeito. No entanto, na antropometria indireta, as medidas podem ser coletadas a partir da cefalometria do perfil do tecido mole 1,3,9-14

Constata-se na literatura, como medidas da cabeça e da face na antropometria médica 47 pontos craniofaciais. No que se menciona às medidas antropométricas orofaciais, a altura do lábio superior (sn-sto) corresponde à distância entre o subnasal (sn) e o ponto mais inferior do lábio superior (sto-estômio), a altura do lábio inferior (sto-gn) corresponde à distância entre o ponto superior do lábio inferior (sto) até o tecido mole do gnátio (gn). A altura do filtro (sn-ls) equivale à distância entre o ponto subnasal (sn) e o labial superior (Is), a altura do terço superior da face (tr-g) corresponde à medida do trichion (tr) à glabela $(\mathrm{g})$; a altura do terço médio da face ( $\mathrm{g}$-sn) corresponde à medida da glabela (g) ao subnasal (sn) e altura do terço inferior da face (sn-gn) corresponde à medida do subnasal (sn) ao gnátio (gn). A distância entre o canto externo do olho (ex) e o cheilion (ch) do lado direito e esquerdo da face corresponde à distância entre esses pontos da face $3,9,10-16$.

Dados de normalidade de medidas antropométricas orofaciais são encontrados na literatura de cirurgia craniomaxilofacial, antropometria e ortodontia, porém, a maioria refere-se a adultos. Buscando contribuir para o conhecimento sobre a existência de uma inter-relação entre as medidas orofaciais e os diferentes tipos faciais, o objetivo do presente estudo foi verificar, em uma amostra de adolescen- tes, se há diferença nas medidas faciais segundo a tipologia facial.

\section{MÉTODOS}

Esse estudo é do tipo transversal, sendo que os dados referentes à tipologia facial foram coletados de prontuários, a partir da documentação ortodôntica e cefalometrias, caracterizando-o também como uma pesquisa retrospectiva.

Foram selecionadas, nos arquivos de documentação da Clínica Odontológica Integrada Ltda ME da cidade de Cabo Frio no estado do Rio de Janeiro, análises cefalométricas de Ricketts determinando os tipos faciais (longo, médio e curto) de adolescentes brasileiros, provenientes do estado do Rio de Janeiro, leucodermas, com idades entre 15 e 17 anos, do sexo masculino e feminino.

Para realização desta pesquisa foram avaliados 39 adolescentes destes arquivos selecionados, sendo que $16(41,03 \%)$ dos adolescentes eram dolicofaciais, $13(33,33 \%)$ eram mesofaciais e 10 $(25,65 \%)$ eram braquifaciais.

Os critérios de inclusão dos adolescentes para este estudo foram: a) ser leucoderma; b) apresentar dentição permanente e; c) ter realizado cefalometria antes do início do tratamento ortodôntico. Os critérios de exclusão foram: a) inexistência de queixa (s) fonoaudiológica(s), independentemente da área (voz, linguagem, motricidade orofacial, e/ou audição, e/ou de histórico de tratamento fonoaudiológico prévio e/ou atual; b) histórico de síndrome e/ ou doença neurológica e/ou úvula bífida.

Todos os participantes foram submetidos à avaliação que constou das seguintes etapas: a) entrevista inicial realizada com os pais ou responsáveis pelos adolescentes, cujo objetivo foi investigar os critérios de inclusão e exclusão dos sujeitos; b) exame clínico das medidas antropométricas orofaciais, obtidas no próprio consultório odontológico, utilizando o paquímetro digital CE ELECTRONIC, fabricado na China. Inicialmente era verificado se os adolescentes eram leucodermas e se encontravam em dentição permanente, estando os mesmos sentados, com os pés apoiados no chão, com a cabeça em posição habitual e com os lábios ocluídos, para essa verificação. Os adolescentes que apresentaram essas características foram seguidos com a coleta dos dados antropométricos.

Foi mostrado a cada sujeito o paquímetro e explicado seu funcionamento, a fim de se obter a familiarização com o instrumento, evitando reações adversas com a musculatura facial durante a obtenção das medidas orofaciais.

Os pontos antropométricos orofaciais foram palpados antes das medidas serem colhidas, para 
a determinação da localização dos mesmos. As medidas antropométricas orofaciais foram obtidas diretamente em cada adolescente por uma das pesquisadoras, na posição frontal, sem forçar suas hastes sobre a pele, duas vezes cada medida, a fim de aumentar a confiabilidade. Calculou-se, posteriormente, a média aritmética das duas medidas obtidas de cada estruturada avaliada. Todas as medidas foram registradas no protocolo de coleta para registro individual de cada medida orofacial (Figura 1).

As medidas orofaciais colhidas foram: altura do lábio superior (do subnasal ao estômio ou sn-sto); altura do lábio inferior (do estômio ao gnátio ou stogn); altura do filtro (do subnasal ao labial superior ou sn-Is); altura do terço superior da face (do trichion a glabela ou tr-g); altura do terço médio da face (da glabela ao subnasal ou g-sn); altura do terço inferior da face (do subnasal ao gnátio ou sn-gn); distância entre o canto externo do olho e o cheilion do lado direito da face (ex-ch); e a distância entre o canto externo do olho e o cheilion do lado esquerdo da face (ex-ch).

Ao final da avaliação de cada sujeito, as luvas cirúrgicas foram inutilizadas e as hastes do paquímetro foram lavadas com água e detergente e desinfetadas com álcool etílico, friccionado com algodão. Os procedimentos com cada adolescente duraram aproximadamente 15 minutos.

Esta pesquisa foi aprovada pelo Comitê de Ética em Pesquisa do CEFAC - Pós-Graduação em Saúde e Educação sob o número 022/08, tendo sido considerada sem risco com necessidade do Termo de Consentimento Livre e Esclarecido. Foi solicitado aos pais ou responsáveis, por intermédio dos sujeitos, o preenchimento deste termo.

Foi realizada a comparação das médias das medidas orofaciais nas distribuições dos três tipos faciais, em adolescentes segundo idade, por meio da análise de variância a um fator. Os dados

\section{Protocolo de coleta de dados}

\section{Dados de identificação}

Nome: Sexo: número:

Data de nascimento: Idade: Data:

\section{Medidas antropométricas orofaciais}

\begin{tabular}{|c|c|c|c|c|}
\hline Região & Estrutura & $\begin{array}{l}\text { Medida I } \\
\text { (em } \mathrm{mm})\end{array}$ & $\begin{array}{l}\text { Medida II } \\
\text { (em } \mathrm{mm})\end{array}$ & $\begin{array}{c}\text { Média } \\
(\mathrm{em} \mathrm{mm})\end{array}$ \\
\hline \multirow{5}{*}{ Facial } & $\begin{array}{l}\text { terço superior } \\
\text { (tr-g) }\end{array}$ & & & \\
\hline & $\begin{array}{c}\text { terço médio } \\
(\mathrm{g}-\mathrm{s} n)\end{array}$ & & & \\
\hline & $\begin{array}{l}\text { terço inferior } \\
\text { (sn-gn) }\end{array}$ & & & \\
\hline & $\begin{array}{l}\text { canto externo do } \\
\text { olho ao cheilion no } \\
\text { lado direito (ex-ch) }\end{array}$ & & & \\
\hline & $\begin{array}{c}\text { canto externo do } \\
\text { olho ao cheilion no } \\
\text { lado esquerdo } \\
\text { (ex-ch) }\end{array}$ & & & \\
\hline \multirow{3}{*}{ Nasolabial } & $\begin{array}{c}\text { lábio superior } \\
\text { (sn-sto) }\end{array}$ & & & \\
\hline & $\begin{array}{c}\text { lábio inferior } \\
\text { (sto-gn) }\end{array}$ & & & \\
\hline & filtro (sn-ls) & & & \\
\hline
\end{tabular}

Figura 1 - Protocolo adaptado de Cattoni ${ }^{14}$ 
obtidos foram analisados estatisticamente por meio dos testes paramétricos ANOVA, teste de Anderson-Darling (gráfico de distribuição de normalidade, sigla $A D$ ) e o teste de Levene (homogeneidade das variâncias).

Ao ser detectada diferença estatisticamente significante entre os tipos faciais, foram feitas as comparações múltiplas utilizando-se o teste de Turkey$H S D$ (honest significant differences).

Foi utilizado Programa SPSS (Statistical Package for Social Sciences), em sua versão V11.5, Minitab 14 e Excel XP, para a obtenção dos resultados. Para cada medida orofacial, foi calculado o intervalo de confiança de $95 \%$. Em todas as análises utilizou-se o nível de significância de 5\%.

\section{RESULTADOS}

Os resultados estão distribuídos nas Tabelas de 1 a 4 , em que a amostra foi caracterizada por meio de análise descritiva dos dados (média, tipo facial, intervalo de confiança e valores mínimos e máximos). Realizou-se a comparação do tipo facial entre a média das medidas dos lábios superior e inferior, filtro, terços superior médio e inferior da face, e distância entre o canto externo do olho e a comissura labial nos lados direito e esquerdo.

Em relação aos lábios, somente para o superior é que houve diferença entre os tipos faciais, sendo o valor dos dolicofaciais maior que o encontrado para os sujeitos mesofaciais (Tabela 1).

Para as medidas de filtro, houve diferença entre as médias, sendo esta entre o grupo dolicofacial e braquifacial $(p=0,030)$, cujo valor desse último foi menor (Tabela 2).

Como mostrado na Tabela 3, houve diferença para o terço superior da face, sendo o valor do tipo mesofacial menor que os demais tipos $(p=<0,001)$ e, no terço inferior, o valor do dolicofacial foi maior que o braquifacial $(p=0,007)$, não havendo diferença entre os grupos para o terço médio da face.

No que se refere aos valores da distância entre o canto externo do olho e a comissura labial em ambos os lados da face (Tabela 4), as médias dos valores, tanto no lado direito quanto no lado esquerdo foram muito próximas, não havendo diferença entre os tipos faciais.

Tabela 1 - Altura do lábio superior e inferior segundo tipologia facial

\begin{tabular}{ccccccc}
\hline \multirow{2}{*}{ Lábio } & \multicolumn{3}{c}{ Superior } & \multicolumn{3}{c}{ Inferior } \\
\cline { 2 - 7 } & Dolicofacial & Mesofacial & Braquifacial & Dolicofacial & Mesofacial & Braquifacial \\
\hline Média & 21,57 & 18,43 & 19,42 & 43,25 & 42,41 & 40,55 \\
Mediana & 21,7 & 18,3 & 19,9 & 43,6 & 40,0 & 40,0 \\
Desvio Padrão & 2,65 & 2,02 & 3,02 & 3,36 & 4,10 & 6,13 \\
Mínimo & 17,0 & 16,4 & 14,8 & 37,5 & 37,9 & 34,3 \\
Máximo & 25,7 & 21,6 & 23,7 & 47,9 & 49,3 & 47,7 \\
N & 16 & 13 & 10 & 16 & 13 & 10 \\
IC & 1,30 & 1,10 & 1,87 & 1,65 & 2,23 & 3,80 \\
\hline p-valor & \multicolumn{5}{c}{}
\end{tabular}

Legenda: $\mathrm{N}=$ número da amostra; IC=intervalo de confiança; * = estatisticamente significante

Tabela 2 - Altura do Filtro segundo tipologia facial

\begin{tabular}{cccc}
\hline \multirow{2}{*}{ Tipo Facial } & \multicolumn{3}{c}{ Filtro } \\
\cline { 2 - 4 } & Dolicofacial & Mesofacial & Braquifacial \\
\hline Média & 13,96 & 12,65 & 11,71 \\
Mediana & 13,8 & 12,6 & 12,5 \\
Desvio Padrão & 2,43 & 1,30 & 2,21 \\
Mínimo & 10,6 & 10,5 & 7,9 \\
Máximo & 18,7 & 14,8 & 14,2 \\
N & 16 & 13 & 10 \\
IC & 1,19 & 0,70 & 1,37 \\
\hline p-valor & & $0,030^{*}$ &
\end{tabular}

Legenda: $\mathrm{N}=$ número da amostra; $\mathrm{IC}=$ intervalo de confiança; * = estatisticamente significante 
Tabela 3 - Altura dos terços da face segundo tipologia facial

\begin{tabular}{|c|c|c|c|c|c|c|c|c|c|}
\hline \multirow{2}{*}{ Terço } & \multicolumn{3}{|c|}{ Superior } & \multicolumn{3}{|c|}{ Médio } & \multicolumn{3}{|c|}{ Inferior } \\
\hline & Dolicofacial & Mesofacial & Braquifacial & Dolicofacial & Mesofacial & Braquifacial & Dolicofacial & Mesofacial & Braquifacia \\
\hline Média & 57,39 & 47,92 & 55,20 & 62,58 & 61,71 & 63,99 & 67,12 & 63,18 & 61,40 \\
\hline Mediana & 55,6 & 49,1 & 55,2 & 62,7 & 60,7 & 63,2 & 67,4 & 63,1 & 61,5 \\
\hline Desvio Padrão & 6,23 & 3,50 & 4,08 & 3,67 & 3,89 & 3,21 & 4,29 & 3,74 & 5,51 \\
\hline Mínimo & 48,1 & 42,4 & 50,1 & 57,5 & 56,3 & 60,8 & 60,4 & 57,9 & 54,0 \\
\hline Máximo & 69,0 & 53,5 & 61,5 & 70,0 & 67,4 & 70,0 & 76,2 & 68,6 & 67,6 \\
\hline $\mathrm{N}$ & 16 & 13 & 10 & 16 & 13 & 10 & 16 & 13 & 10 \\
\hline IC & 3,05 & 1,90 & 2,53 & 1,80 & 2,11 & 1,99 & 2,10 & 2,03 & 3,42 \\
\hline $\mathrm{p}$-valor & & $<0,001^{*}$ & & & 0,337 & & & $0,007^{*}$ & \\
\hline
\end{tabular}

Legenda: $\mathrm{N}=$ número de amostra; IC=intervalo de confiança; *= estatisticamente significante

Tabela 4 - Lados da face segundo tipologia facial

\begin{tabular}{ccccccc}
\hline \multirow{2}{*}{ Lado } & \multicolumn{3}{c}{ Direito } & \multicolumn{3}{c}{ Esquerdo } \\
\cline { 2 - 7 } & Dolicofacial & Mesofacial & Braquifacial & Dolicofacial & Mesofacial & Braquifacial \\
\hline Média & 73,35 & 72,70 & 73,51 & 73,41 & 72,66 & 73,26 \\
Mediana & 74,0 & 73,2 & 73,7 & 73,6 & 72,4 & 73,3 \\
Desvio Padrão & 2,39 & 2,04 & 2,44 & 1,98 & 1,66 & 1,80 \\
Mínimo & 67,0 & 68,8 & 69,4 & 68,4 & 70,5 & 70,3 \\
Máximo & 76,0 & 75,3 & 77,2 & 76,9 & 75,1 & 76,8 \\
N & 16 & 13 & 10 & 16 & 13 & 10 \\
IC & 1,17 & 1,11 & 1,51 & 0,97 & 0,90 & 1,11 \\
\hline p-valor & 0,655 & \multicolumn{5}{c}{0,533} \\
\hline
\end{tabular}

Legenda: $\mathrm{N}=$ número da amostra; IC=intervalo de confiança; *= estatisticamente significante

\section{DISCUSSÃO}

Como foi possível observar nesta amostra, para a altura dos lábios em relação à variável tipo facial, constatou-se que somente o lábio superior apresentou diferença entre os tipos dolicofacial e o mesofacial.

Quanto à altura do filtro, em todas as categorias estudadas, a média do grupo dolicofacial foi maior que a encontrada para o braquifacial.

Assim, como observado em outra pesquisa com crianças, os valores tanto de lábio superior quanto de filtro foram maiores para o padrão dolicofacial, mostrando com isso a importância da avaliação morfológica quantitativa considerando a tipologia facial ${ }^{3}$.

Os dados para os terços da face encontrados neste estudo mostram que há diferença entre os tipos de face. Sendo assim, no terço superior a diferença está entre mesofacial com os demais tipos de face, e para o terço inferior a diferença está entre dolicofacial e braquifacial. Ao aplicar esses dados em indivíduos com respiração oral, que tendem a ser face longa ${ }^{8,9}$, deve-se considerar a tipologia facial, uma vez que diferenças foram detectadas nas medidas orofaciais de adolescentes dolicofaciais. Tais dados sobre a morfologia orofacial são importantes tanto para o estabelecimento do planejamento terapêutico como para a determinação do prognóstico.

Verifica-se que os tipos faciais evidenciaram resultados semelhantes para ambos os lados da face nesta amostra, sem diferença significativa. $\mathrm{Na}$ literatura há valores de referência para algumas medidas antropométricas estudadas nessa amostra, evidenciando, por exemplo, que pode haver ou não diferença entre os lados da face, de acordo com o crescimento facial e o aumento de idade, tipo de respiração e padrão mastigatório ${ }^{4,7,16}$.

Alguns estudos referentes ao exame da morfologia orofacial citam que as funções estomatognáticas exigem a participação das estruturas faciais e, por isso, é de suma importância a análise da morfologia orofacial, haja visto que no terço médio da face pode ocorrer o hipodesenvolvimento nos sujeitos com alterações das funções estomatognáticas, além de ser esperado que em crianças com dentadura mista que o terço médio da face seja menor do que os temais terços ${ }^{8,9}$ Por meio da avaliação da morfologia orofacial podem-se reconhecer os diferentes tipos de face como face longa (dolicofacial), face curta (braquifacial) e face média (mesofacial) ${ }^{1}$

A partir dos resultados apresentados, podese descrever as médias das medidas orofaciais 
antropométricas e estabelecer as correlações entre os grupos. Optou-se pela aplicação da metodologia indicada em estudos antropométricos clássicos ${ }^{1,3,12,14}$, nos quais são preconizados: 1 . O uso do paquímetro para a obtenção das medidas orofaciais; 2. a palpação dos pontos antropométricos orofaciais antes da coleta para correta localização dos mesmos; 3. o posicionamento da cabeça na postura habitual e o sujeito sentado à frente do examinador, bem como a manutenção dos lábios ocluídos; e 4. o não pressionamento das pontas do paquímetro sobre a pele ${ }^{8,11}$. Além disso, para aumentar a confiabilidade dos dados, os procedimentos foram realizados somente por um fonoaudiólogo, para não haver diferença entre examinadores. Faz-se importante, também, a realização da coleta de dados para ambos os sexos, assim como foi feita nesta pesquisa.

O conhecimento sobre os procedimentos da Antropometria ${ }^{7,11,14}$ pode complementar o exame físico fonoaudiológico, fornecendo dados objetivos e facilmente reproduzíveis, aumentado a precisão do diagnóstico e posteriores avaliações. A Antropometria pode, assim, ajudar no embasamento e documentação do trabalho fonoaudiológico.

As medidas antropométricas orofaciais abordadas neste estudo foram propostas por significativas publicações da área, que podem fornecer dados sobre a morfologia da face nas regiões de interesse para avaliação e tratamento fonoaudiológico, pois as medidas orofaciais escolhidas neste estudo podem estar comprometidas nos indivíduos que apresentam distúrbios miofuncionais orofaciais.
Acredita-se que o fonoaudiólogo tem a capacitação necessária para a realização das avaliações da morfologia orofacial, que facilitam a prática clínica e o trabalho interdisciplinar. Por outro lado, somente por meio da pesquisa científica, respaldada na atuação clínica, poderá promover sua credibilidade junto aos profissionais da saúde e à população em geral.

Para o diagnóstico, planejamento e prognóstico do tratamento miofuncional, é de suma importância o exame da morfologia orofacial pré e pós-tratamento, considerando a tipologia facial, porque permite ao fonoaudiólogo avaliar objetivamente os resultados obtidos. O incentivo a essa prática e o adequado uso dos instrumentos são a base para documentar os dados de forma precisa, aumentando a objetividade dos procedimentos fonoaudiológicos.

\section{CONCLUSÃO}

Em uma amostra de adolescentes leucodermas e sem histórico de distúrbio miofuncional, a medida de altura de lábio superior, filtro, terço superior e inferior da face mostrou-se diferente segundo os tipos faciais, sendo a altura do lábio superior maior no dolicofacial que no mesofacial, a altura do filtro maior no dolicofacial que no braquifacial, o terço superior da face maior no mesofacial comparado aos demais tipos e o terço inferior maior no dolicofacial que no braquifacial. Para a medida de altura de lábio inferior e terço médio da face, bem como lados direito e esquerdo da face não houve diferença de acordo com o tipo facial.

\begin{abstract}
Purpose: to check if there is any difference among the orofacial measurements, according to the facial typology. Methods: 39 leukoderm teenagers, aged between 15-17-year old of both genders, with permanent dentition and with no history of speech and swallowing disorders or treatment. Using a digital instrument (CE ELECTRONIC), measures we carried out concerning length of the upper and lower lip and philtrum, thirds of the face as well of the sides of the face measures. Results: considering the facial topology variable, there was a difference as for the length of the upper lip and philtrum and superior and inferior thirds of the face according to the facial type. We did not observe difference among the facial types as for the length of the lower lip and middle thirds of the face and the measures on both sides of the face. Conclusion: in the studied sample, the length of upper lip was bigger in the long face than in the medium face, the length of the philtrum was bigger in the long face than in the short face, the superior thirds of the face was bigger in the medium face than in the others types and inferior thirds was bigger in the long face than in the short face.
\end{abstract}

KEYWORDS: Face; Anthropometry; Measures; Adolescent; Cephalometry; Morphology 


\section{REFERÊNCIAS}

1. Jardini RSR. Avaliação facial a partir da relação eletromiográfica e antropométrica do músculo bucinador. Rev Soc Bras Fonoaudiol. 2005; 10(3):161-8.

2. Bianchini EMG. A cefalometria nas alterações miofuncionais orais: diagnóstico e tratamento fonoaudiológico. Carapicuiba: Pró-Fono; 2002.

3. Daenecke S, Bianchini EMG, Silva APBV. Medidas antropométricas de comprimento de lábio superior e filtro. Pró-Fono. 2006; 18(3):249-58.

4. Gruber KC, Ferreira FV, Cotrim-Ferreira FA, Scavone Júnior H, Carvalho PEG, Quaglio CL. Incidência dos tipos faciais de Ricketts (dolicofacial, mesofacial e braquifacial) nas maloclusões de classe I e classe II divisão 1a . J Bras Ortodon Ortop Facial. 2006; 11(62):180-98.

5. Lopes LD. Tipologia Facial. In: Gonzáles NZT, Lopes LD. Fonoaudiologia e ortopedia maxilar na reabilitação orofacial: tratamento precoce e preventivo terapia miofuncional. São Paulo: Santos; 2000. p. 29-31.

6. Jung MH, Yang WS, Naham DS. Effects of upper lip closing force on craniofacial structures. Am J Orthod Dentofac Orthop. 2003; 123(1):58-63.

7. Farkas LG, Katic MJ, Forrest CR. Anthropometric proportion indices in the craniofacial regions of 73 patients with forms of isolated coronal synostosis. Ann Plast Surg. 2005; 55(5):495-9.

8. Cattoni DM. Estudo sobre as medidas faciais em crianças: correlações com alteração de mordida e uso de aparelho ortodôntico. Rev Soc Bras Fonoaudiol. 2005; 10(1):1-6.
9. Sies ML, Farias SR, Vieira MM. Respiração oral: relação entre o tipo facial e a oclusão dentária em adolescentes. Rev Soc Bras Fonoaudiol. 2007; 12(3):191-8.

10. Cattoni DM, Fernandes FDM, Marchesan IQ, Latorre MRDO. Medidas antropométricas faciais em crianças segundo períodos da dentição mista. Rev CEFAC. 2003; 5(1):21-9.

11. Cattoni DM. O uso do paquímetro na avaliação da morfologia orofacial. Rev Soc Bras Fonoaudiol. 2006; 11(1):52-8.

12. Parro FM, Toledo MR, Gomes ICD, Marchesan IQ. Diferenças antropométricas entre mulheres brancas e negras após crescimento puberal. Rev CEFAC. 2005; 7(4):459-65.

13. Reis SAB, Abrão J, Capelozza Filho L, Claro CAA. Análise facial numérica do perfil de brasileiros Padrão I. Rev Dental Press Ortodon Ortop Facial. 2006; 11(6):24-34.

14. Cattoni DM. O uso do paquímetro na motricidade orofacial: procedimentos de avaliação. Barueri: PróFono; 2006.

15. Farkas LG, Forrest CR, Phillips JH. Comparison of the morphology of the "cleft face" and the normal face: defining the anthropometric differences. J Craniofac Surg. 2000; 11(2):76-82.

16. Ozdemir MB, Ilgaz A, Dilek A, Ayten $H$, Esat A. Describing normal variations of head and face by using standard measurement and craniofacial variability index (CVI) in seven-year-old normal children. J Craniofac Surg. 2007; 18(3):470-4.

DOI: 10.1590/S1516-18462009005000058

RECEBIDO EM: 04/02/2009

ACEITO EM: 30/09/2009

Endereço para correspondência:

Sheila Pereira de Castro Guedes

Rua Geraldo Martins, 201/1404-B

Niterói - RJ

CEP: 24220-380

E-mail: sheilabrandel@yahaoo.com.br 